\title{
Estado actual y perspectivas de la imagen molecular PET en México
}

\author{
Miguel Ángel Ávila-Rodríguez, ${ }^{1 *}$ Belén Rivera-Bravo, ${ }^{2}$ Nora E. Kerik-Rotenberg, ${ }^{3}$ Enrique Vallejo, ${ }^{4}$ \\ Michel Herranz-Carnero ${ }^{5}$ y Christian Buelna-Cano ${ }^{4}$ \\ 'Universidad Nacional Autónoma de México, Facultad de Medicina, Unidad Radiofarmacia-Ciclotrón, Ciudad de México, México; ${ }^{2}$ Universidad \\ Nacional Autónoma de México, Facultad de Medicina, Unidad PET/CT, Ciudad de México, México; ${ }^{3}$ nstituto Nacional de Neurología y Neurocirugía, \\ Unidad de Imagen Molecular PET/CT, Ciudad de México, México; ${ }^{4}$ Centro Médico ABC, Centro Cardiovascular e Imagen Cardiovascular, Ciudad \\ de México, México; ${ }^{5}$ Servicio Gallego de Salud, Imagen Molecular en Oncología, Galicia, España
}

\section{Resumen}

La tomografía por emisión de positrones (PET) es una técnica de diagnóstico médico mediante la cual se obtienen imágenes funcionales a partir de registrar la biodistribución espacio-temporal de radiofármacos específicos dirigidos a blancos moleculares específicos, proveyendo información bioquímica a nivel molecular. A principios de la primera década de este siglo XXI, la Facultad de Medicina de la Universidad Nacional Autónoma de México implementó esta técnica de diagnóstico en México, convirtiéndose en pionera en aplicaciones PET en el país y Latinoamérica. Casi dos décadas después, la PET se ha convertido en una herramienta esencial en la clínica médica. En este artículo se describen los antecedentes, el estado actual, las perspectivas de la imagen molecular PET en México y el impacto que ha tenido en el manejo de pacientes con enfermedades oncológicas, neurológicas y cardiológicas.

PALABRAS CLAVE: Tomografía por emisión de positrones. PET en México. Imagen molecular. Imágenes funcionales.

\begin{abstract}
Positron-emission tomography (PET) is a medical diagnostic technique by means of which functional images are obtained by recording the spatio-temporal biodistribution of specific radiopharmaceuticals targeted at specific molecular objectives, which provides biochemical information at the molecular level. Early in the first decade of this $21^{\text {st }}$ century, the Faculty of Medicine of the National Autonomous University of Mexico acquired the technology to implement this diagnostic technique in Mexico, thus becoming a pioneer in PET applications in the country and in Latin America. Almost two decades after its implementation in Mexico, PET has become an essential tool in medical clinics. This article describes the background, current state and perspectives of PET molecular imaging in Mexico, and the impact it has had on the management of patients with oncological, neurological and heart diseases.
\end{abstract}

KEY WORDS: Positron-emission tomography. PET in Mexico. Molecular imaging. Functional images.

Correspondencia:

*Miguel Ángel Ávila-Rodríguez

E-mail: avilarod@uwalumni.com
Fecha de recepción: 06-05-2019

Fecha de aceptación: 27-05-2019

DOI: 10.24875/GMM.19005257
Gac Med Mex. 2019;155:436-444

Disponible en PubMed www.gacetamedicademexico.com 


\title{
Imagen molecular PET y radiofármacos
}

\author{
Miguel Ángel Ávila-Rodríguez
}

La imagen molecular PET (positron emission tomography) es una técnica de diagnóstico médico por imagen que provee información bioquímica o funcional a nivel molecular y celular de procesos biológicos. Desarrollada a mediados de la década de 1970, fue introducida en México por la Facultad de Medicina de la Universidad Nacional Autónoma de México a principios del 2000, cuando esta institución adquirió un ciclotrón y una cámara PET y creó la Unidad PET-Ciclotrón, convirtiéndose en pionera de PET en México.

\section{Imagen molecular PE en México}

Actualmente, en México hay ocho ciclotrones en operación (Tabla 1), la mitad de ellos en la Ciudad de México, y solo dos pertenencen a instituciones públicas: el de la Universidad Nacional Autónoma de México y el del Instituto Nacional de Cancerología, que entró en operación en el 2017. En cuanto a equipos PET/CT (computer tomography) hay ya 50 en el país: 21 en la Ciudad de México y los demás distribuidos en el resto de los estados (Figura 1). Diez equipos $\mathrm{PET} / \mathrm{CT}$ pertenecen a instituciones públicas, dos a universidades (Universidad Nacional Autónoma de México y la Universidad Autónoma de Nuevo León), uno a una institución de asistencia privada (Hospital Infantil Teletón de Oncología, Querétaro) y 37 a instituciones o empresas privadas.

A casi dos décadas de haber implementado esta técnica de diagnóstico en México se han tenido avances positivos, sin embargo, su uso está todavía muy centralizado y el norte y sureste del país prácticamente no disponen de esta tecnología. La Organización Mundial de la Salud recomienda un indicador de cobertura de dos equipos PET/CT por cada millón de habitantes, indicador que se cumple con creces en países desarrollados. Con una población de aproximadamente 120 millones, México debería contar con 240 equipos.

No existen estadísticas en cuanto al número de estudios PET/CT que se realizan en México, si bien una estimación propia indica aproximadamente 50 mil anuales, de tal forma que anualmente se realizan aproximadamente 400 estudios PET/CT por millón de habitantes, considerando una población de 120 millones, número muy bajo comparado con los de países del primer mundo (5800 en Estados Unidos, 4400 en
Tabla 1. Ciclotrones en operación en México, mayo de 2019

\begin{tabular}{|c|c|c|c|}
\hline Institución/empresa & Sector & Marca, Energía & Ciudad \\
\hline $\begin{array}{l}\text { Facultad de Medicina, } \\
\text { UNAM }\end{array}$ & \multirow[t]{2}{*}{ Público } & $\begin{array}{l}\text { Siemens, } \\
11 \mathrm{MeV} p\end{array}$ & \multirow[t]{4}{*}{$\begin{array}{l}\text { Ciudad de } \\
\text { México }\end{array}$} \\
\hline $\begin{array}{l}\text { Instituto Nacional de } \\
\text { Cancerología }\end{array}$ & & IBA, $18 \mathrm{MeV} p$ & \\
\hline $\begin{array}{l}\text { Hospital Ángeles } \\
\text { Pedregal }\end{array}$ & \multirow[t]{6}{*}{ Privado } & $\begin{array}{l}\text { Siemens, } \\
11 \mathrm{MeV} p\end{array}$ & \\
\hline $\begin{array}{l}\text { Medidores Industriales } \\
\text { y Médicos S. A de C. V. }\end{array}$ & & GE, 16.5 MeV p & \\
\hline OCA Hospital & & GE, 9.6 MeV p & \multirow{2}{*}{$\begin{array}{l}\text { Monterrey, } \\
\text { Nuevo León }\end{array}$} \\
\hline Doctors Hospital & & IBA, $18 \mathrm{MeV} p$ & \\
\hline Guadalajara PET & & GE, $16.5 \mathrm{MeV} p$ & $\begin{array}{l}\text { Zapopan, } \\
\text { Jalisco }\end{array}$ \\
\hline JUAMA & & $\begin{array}{l}\text { Siemens, } \\
11 \mathrm{MeV} p\end{array}$ & $\begin{array}{l}\text { León, } \\
\text { Guanajuato }\end{array}$ \\
\hline
\end{tabular}

UNAM = Universidad Nacional Autónoma de México.

Japón, 1400 en Europa y 1150 en Canadá). ${ }^{1}$ El costo de los estudios PET/CT es elevado dado que la tecnología que se requiere para realizarlos es costosa, si bien es más costoso no contar con ella.

\section{Impacto de la imagen molecular PET}

La imagen molecular PET se ha convertido en una herramienta clínica indispensable para el mejor manejo de pacientes. Particularmente en oncología ha demostrado tener un impacto importante, debido a que ayuda a seleccionar los tratamientos con las mejores probabilidades de éxito dependiendo de las características de la enfermedad de cada paciente, lo que encamina a la medicina personalizada o de precisión, en la que se trata al paciente no a la enfermedad.

Mediante el uso de la imagen molecular PET es posible reducir los costos, directos o indirectos, asociados con procedimientos terapéuticos equivocados, no efectivos o innecesarios. Para que la imagen molecular PET sea costo-efectiva o rentable, debe ser diagnósticamente precisa y efectiva para mejorar el manejo clínico de los pacientes, sin aumentar el costo del tratamiento. En un protocolo realizado en un hospital en España en el que se incluyeron 105 pacientes con diferentes tipos de cáncer, los estudios PET/CT tuvieron influencia en el manejo clínico de 104/105 


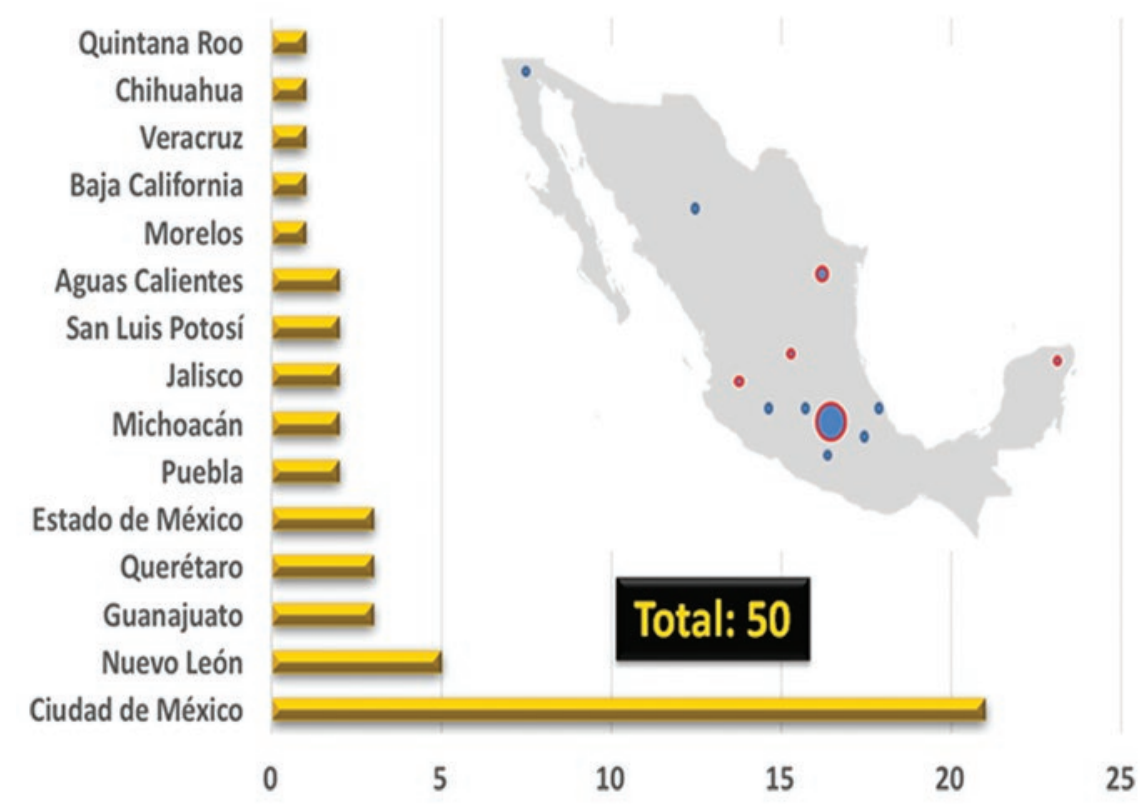

Figura 1. Distribución de equipos PET/CT en México, diciembre de 2018.

pacientes. ${ }^{2}$ En la mitad de los pacientes $(47.8 \%)$ se modificó la estrategia terapéutica y en la otra mitad $(51.3 \%)$ se reafirmó la decisión terapéutica. En ese protocolo también se realizó un estudio de costo-efectividad del PET/CT: se registraron ahorros considerables en procedimientos terapéuticos o de diagnóstico evitados, superiores al costo de los estudios PET/CT realizados. Considerando todos los pacientes, el cociente ahorro/costo fue de 1.33. En cáncer de próstata, cáncer de pulmón y melanoma, el cociente ahorro/ costo fue de $2.06,1.98$ y 1.95 , respectivamente. ${ }^{2}$

Incluso antes del desarrollo de los equipos híbridos PET/CT, en un protocolo clínico realizado en 1996 en el que se incluyeron 207 pacientes con diferentes tipos de cáncer se reportó que el costo de los estudios PET representaba aproximadamente la mitad del costo de los procedimientos terapéuticos evitados y aproximadamente una cuarta parte si el estudio PET reemplazaba un estudio $\mathrm{CT}$. $^{3}$

\section{Radiofármacos}

Los radiofármacos son compuestos que contienen átomos radiactivos en su estructura química y que por su carácter farmacéutico, cantidad y calidad de radiación emitida son adecuados para su administración en seres humanos con fines de diagnóstico o tratamiento. Los radiofármacos tienen dos componentes principales, una molécula de interés biológico que define la ruta metabólica y un radioisótopo que por su característica de emitir radiación permite su detección externa. La característica de los radiofármacos utilizados en PET es que están marcados con un emisor de positrones y que por su vida media corta son producidos a demanda, todos los días, mediante reacciones nucleares en un acelerador de partículas tipo ciclotrón.

El éxito de la imagen molecular PET en México ha ido de la mano de la radiofarmacia. El radiofármaco más utilizado para estudios PET es la glucosa marcada con flúor-18, comúnmente conocido como FDG, el cual permite evaluar el metabolismo de la glucosa y tiene aplicaciones muy diversas; $90 \%$ de los estudios PET que se realizan en el mundo se hacen con FDG. Sin embargo, existen otros radiofármacos, muchos de ellos dirigidos a blancos moleculares específicos, que permiten evaluar procesos fisiológicos y fisiopatológicos a nivel molecular, ofreciendo información más específica de la que ofrece el FDG.

La Unidad Radiofarmacia-Ciclotrón de la Facultad de Medicina de la Universidad Nacional Autónoma de México produce una amplia gama de radiofármacos, la mayoría para estudios oncológicos. Sin embargo, en años recientes se implementó la producción de radiofármacos para el estudio de enfermedades relacionadas con la disfunción de receptores y neurotransmisores. Algunos de los utilizados en oncología tienen también aplicaciones en cardiología, pero el estudio PET más común en cardiología es la perfusión cardiaca. En las siguientes secciones de este simposio se describen las aplicaciones en oncología, neurología y cardiología que se llevan a cabo en México, así como los radiofármacos específicos utilizados en ellas. 


\section{Aplicaciones en oncología}

\section{Belén Rivera-Bravo y Michel Herranz-Carnero}

La Sociedad de Medicina Nuclear e Imagen Molecular de Estados Unidos define la imagen molecular como "la visualización, caracterización y medición de procesos biológicos a nivel molecular y celular en humanos y otros sistemas vivos", ${ }^{4}$ lo que permite divisar las vías metabólicas intracelulares, la expresión de receptores, la síntesis de proteínas, el transporte enzimático, entre otros muchos procesos que suceden al mismo tiempo dentro de las células y en su entorno (ambiente). Los ligandos, moléculas, proteínas, antígenos, receptores, etcétera, que intervienen en estos procesos se convierten en blancos moleculares que pueden marcarse con radioisótopos emisores de positrones para convertirse en radiofármacos, que permiten, mediante la tecnología PET, obtener imágenes y datos para "visualizar, caracterizar y medir" procesos biológicos. ${ }^{4,5}$

Quizá la ruta metabólica más útil en estudios de proliferación y que mejor se conoce es el metabolismo de la glucosa (glucólisis), la cual a su vez es la más utilizada (y la primera empleada) en la PET. En estos estudios, un análogo de la glucosa se marca con ${ }^{18} \mathrm{Flúor}\left({ }^{18} \mathrm{~F}\right)$, convirtiéndose en ${ }^{18} \mathrm{~F}$-fluorodesoxiglucosa (FDG), radiofármaco de gran utilidad en oncología, neurología, cardiología y evaluación de otras patologías, como procesos infecciosos e inflamatorios. ${ }^{6}$ Por su amplio uso, disponibilidad y millones de estudios realizados, este radiofármaco se ha convertido en el recurso por excelencia de la PET/CT.

El equipo de PET/CT permite obtener imágenes fusionadas de ambas modalidades. Es decir, información anatómica que corresponde al estudio de CT e imágenes funcionales o moleculares que resultan del PET. La fusión de ambas imágenes anatómicas y funcionales permite obtener mayor información y precisión diagnóstica que la que se tendría a partir de cada estudio por separado $(1+1=3$, según describió su inventor David Townsend en 1999). ${ }^{7}$ Por tanto, los estudios PET/CT se han convertido en una excelente herramienta diagnóstica no invasiva y en parte fundamental de la imagen molecular.

En cuanto al cáncer, en 2010 Hanahan y Weinberg ${ }^{8}$ describieron los procesos patológicos característicos que suceden en las células y en su entorno durante la transformación oncogénica: proliferación celular sostenida, angiogénesis, hipoxia, alteraciones en la regulación energética, inflamación, entre otros. Estos

Tabla 2. Descripción de los radiofármacos disponibles en México para uso oncológico, su blanco molecular e indicaciones principales

\begin{tabular}{|c|c|c|}
\hline Radiofármaco & Blanco molecular & Indicaciones principales \\
\hline${ }^{18} \mathrm{~F}$-fluorodeoxiglucosa & Metabolismo glucolítico & $\begin{array}{l}\text { Estadificación, restadificación, evaluación de la respuesta al tratamiento, } \\
\text { evaluación de enfermedad residual y recurrencia tumoral, planeación de la } \\
\text { radioterapia, predictor de la respuesta terapéutica o factor pronóstico. }\end{array}$ \\
\hline${ }^{18} \mathrm{~F}$-fluorotimidina & Síntesis de ADN & $\begin{array}{l}\text { Proliferación celular, indicador de respuesta temprana al tratamiento, } \\
\text { diferenciando tejido inflamatorio de tumor viable. }\end{array}$ \\
\hline $\begin{array}{l}{ }^{11} \mathrm{C} \text {-metionina } \\
{ }^{18} \mathrm{~F} \text {-fluoroetiltirosina }\end{array}$ & $\begin{array}{l}\text { Síntesis y transporte de } \\
\text { aminoácidos }\end{array}$ & $\begin{array}{l}\text { Evaluar el grado tumoral, determinar recidiva versus radionecorsis y respuesta a } \\
\text { tratamiento. }\end{array}$ \\
\hline${ }^{18} \mathrm{~F}$-fluorodopamina & Síntesis de dopamina & Evaluación de tumores neuroendocrinos funcionales. \\
\hline${ }^{18} \mathrm{~F}$-fluoruro de sodio & Metabolismo óseo & Actividad osteoneogénica incrementada. \\
\hline${ }^{18} \mathrm{~F}-\mathrm{PSMA}$ & $\begin{array}{l}\text { Antígeno prostático específico } \\
\text { de membrana }\end{array}$ & Estadificación y evaluación de recurrencia bioquímica de cáncer de próstata. \\
\hline${ }^{68} \mathrm{Ga}-\mathrm{DOTANOC}$ & Receptores de somatostatina & $\begin{array}{l}\text { Expresión de receptores de somatostatina, determinar el grado de desdiferenciación } \\
\text { de tumores neuroendocrinos, predecir y evaluar la respuesta al tratamiento. }\end{array}$ \\
\hline${ }^{18} \mathrm{~F}$-fluoroestradiol & Receptores estrogénicos & Densidad y heterogeneidad de la expresión de receptores. \\
\hline${ }^{18} \mathrm{~F}$-misonidazol & Hipoxia tisular & Predecir la respuesta al tratamiento. Determinar la estrategia terapéutica. \\
\hline${ }^{68} \mathrm{Ga}-\mathrm{RGD}$ & Angiogénesis & $\begin{array}{l}\text { Expresión y localización de la integrina } \alpha_{v} \beta_{3} \text {. Planificación y evaluación de } \\
\text { terapias antiangiogénicas. }\end{array}$ \\
\hline${ }^{11} \mathrm{C}$-acetato & Metabolismo de ácidos grasos & Hepatocarcinoma y evaluación de lesiones renales indeterminadas. \\
\hline
\end{tabular}



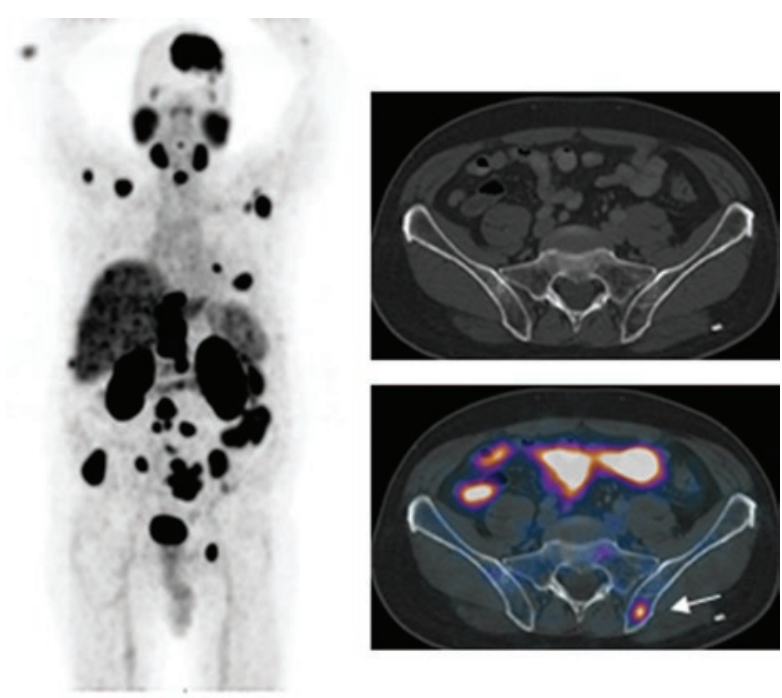

Figura 2. A la izquierda, estudio PET con ${ }^{68} \mathrm{Ga}-\mathrm{PSMA}$, realizado para la estadificación de un paciente con cáncer de próstata. Además de la biodistribución fisiológica del radiofármaco, se observa la gran extensión metastásica ganglionar y ósea de la enfermedad. A la derecha, cortes axiales de tomografía computarizada y PET/CT de la región pélvica de un paciente con cáncer de próstata enviado para evaluación con ${ }^{18} \mathrm{~F}$-PSMA por recurrencia bioquímica. La tomografía computarizada no muestra anormalidades detectables, mientras que en el mismo corte de la fusión PET/CT se observa captación focal del radiofármaco a nivel del iliaco izquierdo (flecha), sin cambios anatómicos detectables, compatible con actividad tumoral del tumor primario conocido.

procesos se convierten en potenciales blancos moleculares que se pueden evaluar mediante la imagen molecular, utilizando radiofármacos específicos, con lo que cada vez se está más cerca del concepto de medicina personalizada, centrada en y para cada paciente específico. En México, actualmente para la evaluación del paciente oncológico se tiene disponible una amplia variedad de radiofármacos (Tabla 2).

La sensibilidad y especificidad de los estudios PET dependen de la patología, es decir, cuanta mayor información disponible sobre las características moleculares del tumor y del contexto clínico del paciente, mejor se podrá seleccionar, y con mayor precisión, el radiofármaco que suministre mayor información sobre la enfermedad (Figura 2).

Un avance que ha proporcionado la imagen molecular es el "teranóstico", término que une terapia y diagnóstico en un mismo concepto y con la misma molécula. Esto significa que el blanco molecular para el diagnóstico es utilizado también para el tratamiento, es decir, el proceso biológico se evalúa mediante $\mathrm{PET} /$ CT, lo que permite su caracterización; posteriormente, el radioligando que se utilizó para el estudio de imagen molecular se une a diferentes átomos radiactivos (emisores alfa o beta) y se administra al paciente para tratamiento. El mismo fármaco para identificar un tumor se emplea para tratatarlo. ${ }^{9}$ Lo anterior es una realidad en México y se efectúa en instituciones públicas y privadas, donde se utiliza el teranóstico para tumores neuroendocrinos y cáncer de próstata.

Otro avance que ofrece la imagen molecular y que revolucionará este campo es la "radiómica", término que se refiere a la conversión de imágenes médicas digitales en minería de datos para generar información objetiva con el fin de mejorar el proceso de toma de decisiones médicas..$^{10}$ La información que provee esta herramienta va más allá de lo que se obtiene mediante el análisis visual, cualitativo o semicuantitativo de las imágenes. Sin duda, la aplicación de algoritmos matemáticos para obtener información objetiva sobre las imágenes cambiará la forma de interpretación actual de los estudios diagnósticos.

La contribución de la imagen molecular a la clínica es muy relevante: no solo se obtiene información a través de estudios no invasivos sobre el comportamiento biológico del tumor y su entorno, sino que además participan en el diagnóstico y la evaluación de la extensión de la enfermedad. En el nuevo paradigma del cáncer puede aportar información precisa a nivel molecular para ayudar en la toma de mejores decisiones terapéuticas y evaluar la respuesta.

Actualmente se dispone de herramientas objetivas y específicas que proporcionan información biológica sobre el comportamiento tumoral, con las que se puede diferenciar entre pacientes que con el mismo diagnóstico, enfermedad, estadio clínico e igual tratamiento, tienen pronósticos distintos. Ahora se sabe que este comportamiento se debe a que las características moleculares de las enfermedades son completamente distintas, por la fisiología y el metabolismo del tumor o su ambiente. Esa información únicamente se puede evidenciar mediante la imagen molecular y con ello contribuir a hacer realidad la medicina personalizada.

Entre los retos actuales de la imagen molecular $\mathrm{PET}$, sin duda los más importantes son:

- La difusión de su utilidad y beneficios en la toma de decisiones clínicas.

- La accesibilidad al método.

- El desarrollo del teranóstico y la radiómica.

Se deben enfrentar activamente estos desafíos y perspectivas para ofrecer al paciente la medicina personalizada que se desea alcanzar.

La Unidad PET/CT de la Facultad de Medicina de la Universidad Nacional Autónoma de México cuenta con equipo de alta tecnología y última generación, el primero en su tipo en Latinoamérica. Esta mejora tecnológica permitirá desarrollar estudios diagnósticos con menor exposición de los pacientes a la radiación, con mayor resolución y mejor capacidad diagnóstica. 


\title{
Aplicaciones en neurología
}

\author{
Nora Estela Kerik-Rotenberg
}

\section{Neurooncología}

Los tumores cerebrales primarios son una causa común de cáncer con pronóstico devastador. Los gliomas constituyen los tumores cerebrales más frecuentes del sistema nervioso central y su diferenciación y graduación histológica son predictivos de la agresividad y pronóstico. El seguimiento del tumor posterior a la cirugía, quimioterapia y particularmente posterior a la radioterapia es difícil con las modalidades de imagen morfológica, ya que estas no tienen la capacidad de diferenciar entre recurrencia tumoral y necrosis posradiación.

La imagen molecular PET provee información metabólica (in vivo) adicional del tumor, tanto para el manejo como para la evaluación de nuevos tratamientos, utilizando marcadores moleculares de metabolismo energético (FDG), de síntesis proteica $\left({ }^{11} \mathrm{C}\right.$-metionina, ${ }^{18} \mathrm{~F}$-fluoroetiltirosina, ${ }^{18} \mathrm{~F}$-fluoro-L-fenilalanina) y ácidos nucleicos ( $\left({ }^{18} \mathrm{~F}\right.$-fluorotimidina), de hipoxia $\left({ }^{18} \mathrm{~F}\right.$-misonidazol) y componentes de membrana celular ( ${ }^{18} \mathrm{~F}$-colina, ${ }^{11} \mathrm{C}$-acetato). ${ }^{11,12}$

Las técnicas de imagen molecular han mostrado tener un enorme potencial para la selección de pacientes candidatos a terapias blanco, así como en la evaluación de la respuesta al tratamiento y, por lo tanto, en la individualización terapéutica.

\section{Demencias}

Desde la década de 1980, la PET se ha considerado uno de los estudios de imagen más precisos para la detección de la enfermedad de Alzheimer. En 2004, la Alzheimer's Association apoyó el uso del FDG-PET en pacientes con demencia o deterioro cognitivo leve o moderado de al menos seis meses de evolución.

El aporte de la PET, entre otras cosas, puede diferenciar la enfermedad de Alzheimer de otras demencias, identificar grupos en riesgo progresivo, acelerar y mejorar la exactitud del diagnóstico precoz y ayudar en el desarrollo de tratamientos que eviten la progresión utilizando marcadores de $\beta$-amiloide y de metabolismo de glucosa.

El radiofármaco más utilizado para PET es el FDG. Su captación refleja el consumo de glucosa regional. Por lo tanto, la degeneración o disfunción neuronal lleva a disminución del metabolismo de la glucosa que se presenta en diferentes patrones cerebrales. ${ }^{13}$ En la enfermedad de Alzheimer, la reducción del índice de consumo de glucosa cerebral es severa, lo que se refleja en la PET como disminución en la función y densidad sináptica, con sensibilidad de $96 \%$ y especificidad de $93 \%$.

Por medio de un estudio PET se puede diferenciar la enfermedad de Alzheimer de otro tipo de demencias, como la frontotemporal, la vascular y la de los cuerpos de Lewy, así como las demencias asociadas con las enfermedades de Huntington y Parkinson.

\section{Trastornos del movimiento}

En las últimas décadas se han desarrollado diversos marcadores para el estudio de los pacientes con enfermedad de Parkinson, para mejorar el diagnóstico diferencial con otros parkinsonismos y para seguimiento de la progresión de la enfermedad. La enfermedad de Parkinson es un trastorno neurodegenerativo caracterizado por pérdida progresiva de neuronas monoaminérgicas, en especial dopaminérgicas. El sistema dopaminérgico puede estudiarse a nivel presináptico y postsináptico..$^{14,15}$ El patrón normal de distribución del radioligando depende de la distribución normal del elemento sináptico por estudiar.

\section{Radioligandos presinápticos}

${ }^{11} \mathrm{C}$-dihidrotetrabenazina, con alta afinidad por el transportador vesicular monoaminérgico tipo 2, permite medir las monoaminas en las vesículas sinápticas (dopamina, serotonina, norepinefrina, histamina) (Figura 3).

${ }^{18} \mathrm{~F}$-fluoro-L-fenilalanina es un precursor de la dopamina y análogo de la levodopa. En la enfermedad de Parkinson, la captación de la fluorodopa en el estriado, especialmente en el putamen, se encuentra reducida, existiendo una correlación con el grado de alteraciones motoras.

\section{Radioligandos postsinápticos}

${ }^{11} \mathrm{C}$-raclopride $0{ }^{18} \mathrm{~F}$-fallypride son los compuestos más utilizados para el estudio de receptores D2. La imagen de receptores de dopamina se caracteriza por 


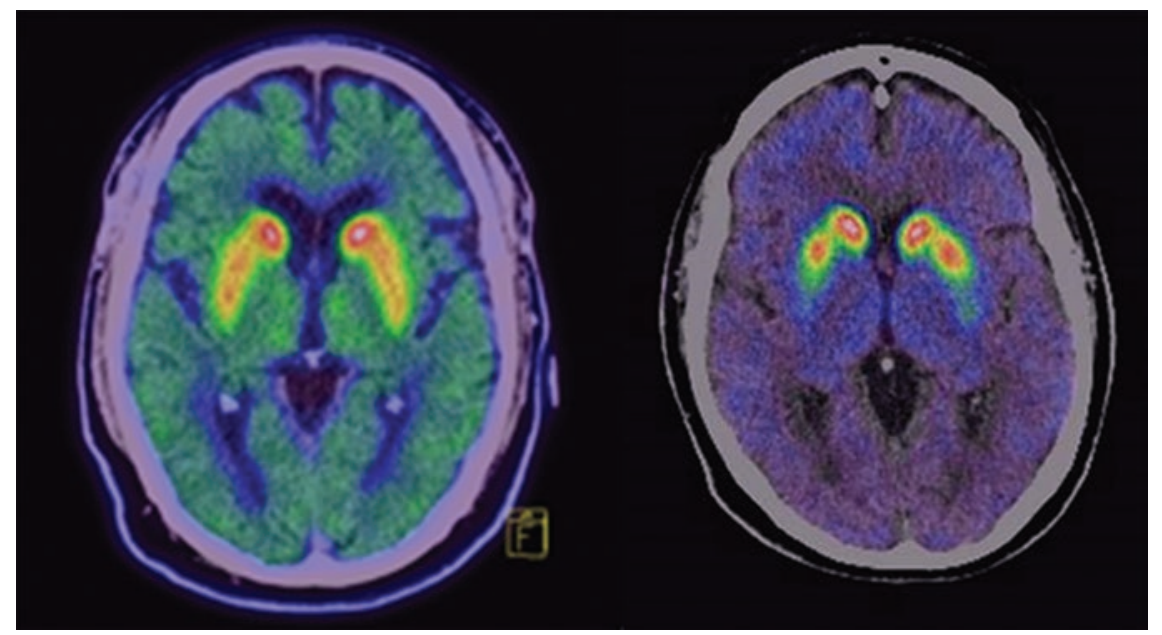

Figura 3. Imagen del sistema dopaminérgico presináptico con ${ }^{11} \mathrm{C}$-dihidrotetrabenazina. A la izquierda un estudio normal y a la derecha, hipocaptación severa nigroestriatal, correspondiente a un patrón "Eagle wing" característico de la enfermedad de Parkinson.

la elevada captación en el estriado debido a la alta densidad de terminales dopaminérgicas existentes en los mismos.

En relación con los síndromes parkinsonianos, estos pertenecen a un grupo de enfermedades neurodegenerativas con pérdida progresiva y crónica de tejido neuronal en los sistemas cognitivo, motor y sensorial. Tales síndromes incluyen enfermedades con trastornos en el movimiento, como la atrofia de múltiples sistemas, degeneración corticobasal y parálisis supranuclear progresiva, enfermedad de Huntington, esclerosis lateral amiotrófica y enfermedad por priones, como la enfermedad de Creutzfeldt-Jakob. Estas enfermedades pueden identificarse con múltiples grados de afectación cortical y del sistema nigroestriatal. La imagen molecular PET permite aproximarse a un diagnóstico diferencial más preciso para evaluar in vivo los mecanismos responsables de los procesos neurodegenerativos.

\section{Epilepsia}

En una proporción significativa de pacientes con epilepsia, $30 \%$ de las crisis parciales complejas son refractarias al tratamiento a pesar de los esfuerzos terapéuticos. La selección de pacientes sin respuesta al tratamiento médico y candidatos a cirugía requiere fundamentalmente de la correcta localización prequirúrgica de la zona epileptógena y de la predicción de las posibles secuelas de la intervención. Actualmente se utiliza PET-FDG para la localización metabólica de la zona epileptógena antes de la cirugía, con una sensibilidad de 80 a $90 \%$ en epilepsia temporal, ${ }^{16} \mathrm{y}$ de 45 a $92 \%$ para la epilepsia extratemporal. ${ }^{17}$ El objetivo de la evaluación es determinar si existe evidencia de un foco epiléptico único, determinar sus vías de diseminación y su relación con una posible lesión estructural.

\section{Neurología}

Una de las aplicaciones actuales de PET/CT es el diagnóstico diferencial de las encefalitis. ${ }^{18}$ Las encefalitis autoinmunes constituyen una nueva categoría de enfermedades inflamatorias del sistema nervioso central mediadas contra el anticuerpo de neurotransmisores o proteínas de la superficie neuronal. Los síndromes clínicos son complejos y se asocian con manifestaciones que varían en función del tipo de anticuerpo asociado. La respuesta autoinmune puede iniciarse por un tumor o infección viral, pero en muchos casos se desconoce. La aplicación de PET-FDG ha sido de suma importancia para el diagnóstico diferencial, seguimiento, evaluación de la terapia y pronóstico del paciente. Proporciona también información a nivel metabólico bioquímico-molecular in vivo, por lo que es una herramienta excelente para valorar el metabolismo cerebral y descartar posibles sitios de malignidad desde etapa temprana. 


\title{
Aplicaciones en cardiología
}

\author{
Enrique Vallejo y Christian Buelna-Cano
}

La PET evalúa y cuantifica con certeza la viabilidad miocárdica, la perfusión miocárdica, la reserva del flujo coronario (RFC), los procesos inflamatorios e infecciosos y el daño tisular. ${ }^{19,20}$

\section{Evaluación de viabilidad miocárdica}

La evaluación de la viabilidad miocárdica está indicada en pacientes con infarto y disfunción sistólica (fracción de expulsión del ventrículo izquierdo [FEVI] $<0.35$ ). El concepto de viabilidad miocárdica fue creado para describir a las células con actividad metabólica y sin función contráctil después del infarto. ${ }^{21}$ La imagen diagnóstica por PET en viabilidad miocárdica es la discordancia entre la perfusión y el metabolismo, es decir, defectos de perfusión miocárdica pero captación de FDG gracias al metabolismo celular. El estudio de la viabilidad miocárdica con PET en pacientes con FEVI $<0.35$ diferencia a los segmentos ventriculares con reserva contráctil (valor predictivo positivo de $85 \%$ ) de los segmentos sin reserva contráctil (valor predictivo negativo de $92 \%$ ) después de la revascularización coronaria. El porcentaje de viabilidad miocárdica necesario para inferir mejoría en la supervivencia con la revascularización coronaria es de $7 \%{ }^{22,23}$

\section{Evaluación de perfusión miocárdica}

La PET ofrece ventajas en comparación con la tomografía computarizada con emisión de fotón único para la evaluación de la perfusión miocárdica, ya que cuantifica el flujo miocárdico global y el flujo regional. Con base en estos parámetros cuantitativos se puede calcular la RFC, que se define como la diferencia entre el flujo miocárdico máximo en estrés y el flujo miocárdico en reposo. ${ }^{24}$ Con estos parámetros, la PET identifica pacientes con placas ateromatosas 0 enfermedad microvascular limitantes de la RFC. La cuantificación de la RFC es precisa y tiene un elevado valor pronóstico en pacientes con sospecha de enfermedad arterial coronaria. ${ }^{25}$ Los valores normales del flujo coronario calculados con ${ }^{13} \mathrm{~N}$-amonia en reposo es de $0.71 \mathrm{~mL} / \mathrm{minuto} / \mathrm{g}$ y en estrés de $2.58 \mathrm{~mL} / \mathrm{minu}$ to/g. El valor normal de la RFC es de 3.54. La RFC $>2$ tiene un alto valor predictivo negativo para descartar enfermedad arterial coronaria de alto riesgo, aun en pacientes con enfermedad trivascular. La RFC global $<1.5$ se asocia con incremento de muerte por causas cardiovasculares. ${ }^{26}$ En pacientes con diabetes mellitus, sin enfermedad arterial coronaria obstructiva, pero con RFC anormal, PET identifica un grupo de pacientes con alto riesgo cardiovascular equivalente al de los pacientes con enfermedad arterial coronaria obstructiva. ${ }^{27}$

\section{Evaluación de inflamación}

La PET cardiaca evalúa la aterosclerosis como un fenómeno inmunoinflamatorio. El proceso de aterosclerosis es producto de la inflamación; inicia con la activación de los monocitos, su diferenciación en macrófagos en el subendotelio vascular, que a su vez se convierten en células espumosas. Estás células forman parte de la placa aterosclerosa, compuesta por un núcleo lipídico que en una compleja respuesta inflamatoria da lugar a microcalcificaciones. Los radiotrazadores de la PET se enfocan en los componentes de la aterosclerosis: células inflamatorias, lípidos y ácidos grados, además de angiogénesis, proteasas, trombosis, apoptosis y microcalcificaciones. ${ }^{28}$

Imágenes con ${ }^{18} \mathrm{FDG}$ y ${ }^{18} \mathrm{~F}$-fluororo de sodio permiten identificar las placas ateromatosas y caracterizar placas vulnerables de alto riesgo. La captación de ${ }^{18} \mathrm{~F}-\mathrm{FDG}$ refleja inflamación y la captación de ${ }^{18} \mathrm{~F}$-fluororo de sodio está relacionada con microcalcificación. ${ }^{29}$

\section{Evaluación de infecciones}

La PET con ${ }^{18} \mathrm{~F}-\mathrm{FDG}$ es útil en el diagnóstico y seguimiento de endocarditis infecciosa, infección de dispositivos intracardiacos, infección de dispositivos de asistencia ventricular e infección de injertos vasculares. La aplicación más frecuente es en pacientes con endocarditis. Esta técnica ha mejorado la sensibilidad diagnóstica en combinación con los criterios de Duke, alcanzando una sensibilidad de $95 \%$, especificidad de $90 \%$, valor predictivo positivo de $91 \%$ y valor predictivo negativo de $95 \%$. Cuando PET con ${ }^{18} \mathrm{~F}-\mathrm{FDG}$ se agrega a los hallazgos ecocardiográficos, el valor diagnóstico se incrementa significativamente (96 versus $65 \%$ ), favoreciendo la intervención temprana y 
evitando complicaciones graves tales como abscesos perivalvulares y bloqueo auriculoventricular. ${ }^{30}$ La medición cuantitativa del valor de captación estándar de ${ }^{18} \mathrm{~F}-\mathrm{FDG}>2$ tiene sensibilidad de $100 \%$ y especificidad de $92 \%$ para identificar endocarditis en válvula protésica. $^{31}$

Las infecciones asociadas con los dispositivos intracardiacos ocasionan elevada morbimortalidad. PET con ${ }^{18} \mathrm{~F}-\mathrm{FDG}$ está indicada cuando el diagnóstico no ha podido ser confirmado con ecocardiografía; tiene sensibilidad y especificidad de 96 y $97 \%$, respectivamente. ${ }^{30}$

\section{Evaluación de daño tisular}

La fisiopatología en la degeneración de las válvulas bioprotésicas actualmente es tema de investigación. La calcificación del tejido valvular es un componente inicial en este proceso y puede ser identificada antes de que aparezcan las manifestaciones clínicas de disfunción valvular. La PET cardiaca con ${ }^{18} \mathrm{~F}$-flúor identifica microcalcificaciones valvulares, apoyando la hipótesis de que con PET es factible detectar degeneración valvular en etapas subclínicas, por lo se considera que en el futuro será una herramienta útil en la búsqueda de nuevos tratamientos para detener el proceso degenerativo valvular. ${ }^{32}$

\section{Bibliografía}

1. Azaiez F, Cantone MC. Radioisotope production. En: Azaiez F, Bracco A Dobes J, Jokinen A, Körner GE, Maj A, et al., editores. Nuclear physics for medicine, Francia: European Science Foundation/Nuclear Physics European Collaboration; 2013.

2. Alemany N, Pérez-De Olaguer J, Bertomeu M, Trampal B. Clinical impact and cost-effectiveness of PET-CT in the management of oncologic patients. España: European Society of Radiology; 2013.

3. Valk PE, Pounds TR, Tesar RD, Hopkins DM, Haseman MK. Cost effectiveness of PET imaging in clinical oncology. Nucl Med Biol. 1996;23:737-743.

4. Delbeke D, Royal HD, Frey KA, Graham MM, Segall GM. SNMI/ABNM joint position statement on optimizing training in nuclear medicine in the era of hybrid imaging. J Nucl Med. 2012;53:1490-1494.

5. Wester HJ. Nuclear imaging probes: from bench to bedside. Clin Can Res. 2007:13:43-48

6. Boellaard R, Delgado-Bolton R, Oyen WJG, Giammarile F, Tastch K, Eschner W, et al. FDG PET/CT: EANM procedure guidelines for tumour imaging: versión 2.0. Eur J Nucl Med Mol Imaging. 2015;42:328-354.
7. Townsend DW. Positron emission tomography/computed tomography. Semin Nucl Med. 2008:38:152-166.

8. Hanahan D, Winberg RA. Hallmarks in cancer: the next generation. Cell. 2011;144:646-674.

9. Yordanova A, Eppard E, Kürpig S, et al. Theranostics in nuclear medicine practice. Onco Targets Ther. 2017;10:4821-4828.

10. Gillies RJ, Kinahan PE, Hricak H. Radiomics: imaging are more than pictures, they are data. Radiology. 2016;278:563-577.

11. Herholz K. Brain tumors: an update on clinical PET research in gliomas. Semin Nucl Med. 2017;47:5-17.

12. Yamamoto $Y$, Nishiyama $Y$, Kimura N, Kameyama R, Kawai N, Hatakeyama $\mathrm{T}$, et al. $11 \mathrm{C}$-acetate $\mathrm{PET}$ in the evaluation of brain glioma: comparison with 11C methionine and 18F FDG PET. Mol Imaging Biol. 2008;10:281-287.

13. Silverman D, editor. PET in the evaluation of Alzheimer's disease and related disorders. EE. UU.: Springer; 2009.

14. Berti V, Pupi A, Mosconi L. PET/CT in diagnosis of movement disorders. Ann N Y Acad Sci. 2011;1228:93-108.

15. Juri $C$, Wanner V. Neuroimágenes en enfermedad de Parkinson: rol de la resonancia magnética, el SPECT y el PET. Rev Med Clin Condes. 2016;27:380-391.

16. Cendes F, Theodore WH, Brinkmann BH, Sulc V, Cascino GD. Neuroimaging of epilepsy. Handb Clin Neurol. 2016;136:985-1014.

17. Kumar $\mathrm{A}$, Chugani $\mathrm{H}$. The role of radionuclide imaging in epilepsy, part 2 : epilepsy syndromes. J Nucl Med. 2013:54:1924-1930.

18. Morbelli S, Arbizu J, Booij J, Chen MK, Chetelat G, Cross DJ, et al. The need of standarization and of large clinical studies in an emerging indication of $\left[{ }^{18} \mathrm{~F}\right]-\mathrm{FDG}$ PET: the autoinmune encephalitis. Eur J Nucl Med Mol Imaging. 2017;44:353-357.

19. Vallejo E. El futuro del PET en cardiología. Arch Cardiol Mex. 2002;72:220-225.

20. Bateman T, Dilsizian V, Beanlands R, DePuey G, Heller G, Wolinsky D. American society of nuclear cardiology and society of nuclear medicine and molecular imaging joint position statement on the clinical indications for myocardial perfusion PET. J Nucl Med. 2016;57:1654-1656.

21. Anevekar N, Chareonthaitawee P, Narula J, Gersh B. Revascularization in patients with severe left ventricular dysfunction. J Am Coll Cardiol. 2016;67:2874-2887.

22. Loffler Al, Kramer CM. Myocardial viability testing to guide coronary revascularization. Intervent Cardiol Clin. 2018:7:355-365.

23. Allman K. Noninvasive assessment myocardial viability: current status and future directions. J Nucl Cardiol. 2013;20:618-637.

24. Pelletier-Galarneau M, Martineau P, El-Fakhri G. Quantification of PET myocardial blood flow. Curr Cardiol Rep. 2019;21:11.

25. Dilsizian V, Chandrashekhar Y, Narula J. Quantitative PET myocardial blood flow. JACC: Cardiovascular Imaging. 2017:10:609-610.

26. Murthy V, Bateman T, Beanlands R, Berman D, Borges S, Chareonthaitawee $P$, et al. Clinical quantification of myocardial blood flow using PET: Joint position paper of the SNMMI cardiovascular council and the ASNC. J Nucl Med. 2018:59:273-293.

27. Murthy V, Naya M, Foster C, Gaber M, Hainer J, Klein J, et al. Association between coronary vascular dysfunction and cardiac mortality in patients with and without diabetes mellitus. Circulation. 2012;126:1858-1868.

28. Flemming PF, Moghbel MC, Gerke O, Alavi A. Evolving role of PET in detecting and characterizing atherosclerosis. PET Clin. 2019;14:197-209.

29. Moghbel M, Al-Zaghal A, Werner T, Mihail C, Flemming P, Alavi A. The role of PET in evaluating atherosclerosis: a critical review. Semin Nucl Med. 2018;48:488-497.

30. Millar BC, De Camargo RA, Alavi A, Moore JE. PET/computed tomography evaluation of infection of the heart. PET Clin. 2019;14:251-269.

31. Swart LE, Gomes A, Scholtens A, Sinha B, Tanis W, Lam M, et al. Improving the diagnostic performance of 18F-Fluorodeoxyglucose positron emission tomography in prosthetic heart valve endocarditis. Circulation. 2018:138:1412-1427.

32. Cartlidge TRG, Doris M, Sellers S, Pawade T, White A, Pessotto R, et al. Detection and prediction of bioprosthetic aortic valve degeneration. J Am Coll Cardiol. 2019;73:1107-1119. 